\title{
Building Intelligent Precise Logistics Service System by Key Technologies of RFID in the Internet of Things
}

\author{
Guofang Kuang ${ }^{1, \mathrm{a}^{*}}$ and Zhenfang Song ${ }^{1}$ \\ ${ }^{1}$ College of Information Technology, Luoyang Normal University, Luoyang, 471934, China \\ akuangguofang2012@163.com
}

Keywords: Internet of things; Precise logistics service; Intelligent; Tag and reader; RFID

\begin{abstract}
This paper describes the analysis and research of new intelligent precise logistics system. The development of intelligent logistics is precise, intelligent, integrated and collaborative features. Then this paper discusses the application and prospect of RFID key technology in intelligent logistics. Another advantage of the RFID technology is that it reduces manpower during inventory taking. The RFID is designed to make the registration of automation products. The paper presents building intelligent precise logistics service system by key technologies of RFID in the Internet of things.
\end{abstract}

\section{Introduction}

With the help of electronic identification system, make the goods in transit by a number and special information to distinguish, tracking and monitoring convenient transit time and place. Also can connect with other systems, and it is for the control of transport, transport, distribution and storage process in logistics. Intelligent Personalized is Analysis function.

The Internet of things is actually a big concept of intelligent logistics. The Internet of things after the development of the industry is the integration of logistics, integrated transportation, integrated warehousing industry and transportation industry, including shipping, shipping, and road transport and so on, and concerned with the production of manufacturing enterprises will benefit. In this new logistics system, on the one hand to improve the efficiency of logistics, on the other hand, the quality of the products also increased, thus the production enterprise production and management standardization, goods in circulation in order to protect the interests of consumers, the inside operation system.

The development of intelligent logistics is precise, intelligent, integrated and collaborative features. The precise source of lean is construction theory in construction industry [1]. Lean construction is designed by the precise planning, design and supply, the whole process of product control and information feedback, construction management method, minimizing waste resources to maximize profit value.

A complete RFID system, is a reader (Reader) and the electronic tag (TAG) is also called transponder (Transponder) and application software system of three parts, the working principle of radio wave energy to the Transponder Reader to launch a specific frequency, is used to drive the Transponder circuit will internal data sent at this time, Reader was sequentially receive to interpret the data to the application, and make corresponding treatment. The paper presents building intelligent precise logistics service system by key technologies of RFID in the Internet of things.

\section{Analysis and Research of New Intelligent Precise Logistics System}

Under the new logistics system, when we put the intelligent logistics distribution center back network system, intelligent distribution network visualization management and full automation together, creates a wisdom logistics information platform. Currently, the first part of the Internet of things has begun to explore the construction of the logistics information platform in the Internet of things.

Intelligent can make real-time information collection of future logistics system, and the use of Internet of things system processing, to provide quality information and advisory services to end users, provide the best strategy for logistics enterprises. There is a need to explain, to provide more support 
network for intelligent processing of intelligent logistics, in addition to using the ERP commercial software is integrated planning, management and decision support, intelligent logistics future should pay more attention to the use of complex equipment and network service more intelligent.

The perceptual layer is equivalent to the human eye and skin nerve endings, it is the source of things for object recognition and collecting information, its main function is to identify objects, collecting information, and pass out information [2].

A major feature of JTAV is the globalization of read and writes identification equipment deployment architecture (Global Interrogator Infrastructure). The novelty lies in between the concept, headed by the United States and NATO members do not have to worry about the country and region is different, can use any internal RFID reading and writing device for effective identification of tags, and the tags are stored in data transfer to the label owner. This will greatly save time and resources, as is shown by equation (1) [3].

$$
\left\{\begin{array}{r}
a=\frac{n \sum_{i=1}^{n} x_{i} y_{i}-\sum_{i=1}^{n} x_{i} y_{i}}{n \sum_{i=1}^{n} x_{i}{ }^{2}-\left(\sum_{i=1}^{n} x_{i}\right)} \\
b=\frac{1}{n} \sum_{i=1}^{n} y_{i}-\frac{a}{n} \sum_{i=1}^{n} x_{i}
\end{array}\right.
$$

With the advent of the Internet of things, the logistics industry will usher in a new development opportunity. The concept of things, the first is called in the logistics industry. In order to overcome the disadvantages of electronic logistics, modern logistics system to use information generating apparatus, a vast network of radio frequency identification devices, such as sensors or global positioning system and various device combined with Internet and form, and can realize the intelligent management of the logistics network flow in the instrumented.

RA kind of open scalable networking middleware platform, supporting different manufacturers, different types, different communication modes and different communication protocols, different data format "networking devices, the unique" two collection "technology, the terminal equipment through the data of" two collection ", that is to packet data analysis and conversion, output standard data, as is shown by equation (2).

$$
\begin{aligned}
& p(a(k) \mid s(\kappa))=\frac{1}{(2 \pi)^{q / 2}\left|\Sigma_{\varepsilon(k)}\right|^{1 / 2}} \exp \left[-1 / 2(a(k)-\beta(k) s(k)-\alpha(k))^{\mathrm{T}}\right. \\
& \left.\Sigma_{\varepsilon(k)}^{-1}(a(k)-\beta(\kappa) s(\kappa)-\alpha(\kappa))\right]
\end{aligned}
$$

Many supplies even are ordering. In addition, many materials piled up have not provided a system and service management good, can not play the utility and abandoned [4]. The urgent need for an effective logistics management can track the transport of goods of the technology, known as the in transit visibility (In Transit Asset Visibility). A very important and effective management of the logistics system is to realize the visualization technology. First of all, it can be positioned supplies accurate position, reduce the goods packing, ensure the container or container according to a timely manner to the user, to avoid duplication of supply and waste, improve the efficiency and accuracy of the logistic supply.

Because of the actual object in the process of moving, all links are in motion and loose state, often with the mobile entity in the space and time changes, the results of the impact of the information availability and sharing. Radio frequency identification technology is a new technology which can effectively solve the problem of the input / output of business operation data, the control and tracking of business process, and reduce the error rate.

The reader is placed in the warehouse import and export [5]. When an item into the library, automatic identification tag reader on the items of information, and to store information management system connected; when the goods out of the library, by the same reader automatically identify items of 
information, and transmitted to the management system, by the system of information processing of a library.

In this way, each product can be identified, in the global range positioning, tracking. This will make the whole logistics field into a large network, called "EPC Internet of things". EPC is the dream of all logistics enterprises are added to the network, and use the unified format interactive information. With the EPC network, the global logistics enterprises have an effective means of goods flow and information flow together, and can realize the globalization of electronic logistics.

\section{Application and Prospect of RFID Key Technology in Intelligent Logistics}

RFID does not need to read precision with fixed size and printing quality of paper, more suited to the miniaturization and diverse patterns of development, to facilitate the embedded or attached in different shapes and types of products, anti pollution ability and durability, is the carrier of the traditional bar code paper, so vulnerable to pollution, but RFID has very strong resistant to water, oil and chemical substances [6]. In addition, because the bar code is attached to the plastic bags or cartons, so are particularly vulnerable to damage; RFID is the chip data volume will exist, so free from fouling.

Due to cost constraints, very limited storage space RFID label, and some are even only for unique identification. The RFID tag has some limitations in computing power and power consumption. At the same time tag does not have its own security ability enough, so will cause some illegal communicate with tags, or tampering, remove the label information. So the label of the security and it is integrity, availability, authenticity, validity in the protection mechanism of the trusted enough to guarantee.

The RFID tag will be a large number of sales for the supply chain terminal, especially in the supermarket, RFID tags from the tracking process of artificial intervention, can generate highly accurate business data, so it has great appeal [7]. At present, the world's retail giant WAL-MART is using RFID technology to eliminate bar code, in order to further improve the efficiency of the retail sector, as is shown by equation (3).

$$
\Gamma=\left[\begin{array}{c}
0.5 * t s * t s \\
t s \\
0
\end{array}\right]
$$

Overweight, after sampling, car of weight in RFID unmanned vehicle scale. The specific process, system of vehicles entering in the perception, start the scanning system, scan card, swept to the card, wait for the scale data after the data is written to the stable weight [8]. The sign RFID handheld configured for RFID coal, coal field personnel and vehicle information card at the time of discharge, and operation of PDA, input sign / buckle weight information. Data will be written to the database in real-time through wireless LAN.

Although the technology needs to be improved in the RFID tag technology has become more mature, but in general the product technology is not mature enough, there is a high rate of error (RFID misread the ratio is sometimes as high as $20 \%$ ). A study conducted by Auto-ID Center found that even with a double label, the tray with the RFID label had a $3 \%$ failure rate, while the individual labeled tray had only about $78 \%$ of the information available.

Tag and reader layer communicates through electromagnetic wave using radio frequency signal, without any physical contact and visible in the process of communication, the Internet of things perceived node layer and equipment generally exists in the open environment, the node energy and equipment, processing capabilities and communication range, not to the high strength encryption operation, the to provide flexibility and convenience at the same time to the application of data acquisition system, but also to make the message the lack of safety protection ability of the complex.

In the distribution process, the use of radio frequency identification technology can greatly accelerate the speed of distribution, improve the efficiency and accuracy of picking and distribution process, and can reduce the probability of artificial participation, reduce distribution costs [9]. All the goods in the 
paste RFID tags into the central distribution center, through the entrance pallet installation reader, all the labels on the boxes to read the contents of the tray.

With much digital identification technology is development; RFID also suffers from a lack of interoperability standard problems. However, after the scientist's efforts, the situation has improved, but each method between cost and ability has the obvious difference, so it does not achieve unity in a short time. Today, RFID "label" technology is very advanced, costs have dropped sharply. The main technical field still needs to be improved are the comprehensive commercial card reader and antenna.

\section{Building Intelligent Precise Logistics Service System by Key Technologies of RFID in the Internet of Things}

Another advantage of the RFID technology is that it reduces manpower during inventory taking. The RFID is designed to make the registration of automation products; inventory does not need to manually check or scan barcodes, more quickly and accurately, and reduce the loss. RFID solutions provide accurate information about the inventory situation, managers can quickly identify and correct the low efficiency of the operation, so as to achieve rapid delivery, and minimize the cost of storage.

Build up the communication between reader and tags via a wireless signal channel, reader to send electromagnetic signal through the antenna, electromagnetic signals carry the reader to tag the inquiry instruction [10]. When the label in the reader the scope of work, the label will receive instruction data and energy from the electromagnetic signal, and the label and data to transmit electromagnetic signals to the reader form according to the instructions, or according to the reader's instruction rewriting stored in the RFID tag data. The reader can receive data sent to the RFID tag or label to send data, and can carry on the docking with the background server communication network through a standard interface, realizing the communication data transmission, as is shown by equation (4).

$$
h_{M H D}(A, B)=\frac{1}{N_{A}} \sum_{a_{i} \in A} d_{B}\left(a_{i}\right)
$$

From geographical coverage, JTAV is the world's largest RFID network, the network using active RFID tags and GPS tracking of military supplies, improve the military logistics. It can read the RFID information in 400 locations in 40 countries, including the port, military bases and the world railway terminal. JTAV can track 270000 cargo containers, and even can be real time satellite positioning by the container ships. RFID labels per hour are reporting their information, at the same time; JTAV is also carried out on their track.

The application of RFID in the management of container tracking has the characteristics of long distance, high speed and low cost, so it is the best choice to realize the container and pallet tracking RFID. For the transportation of bulk goods, the most ideal mode of transport is, of course, container transport.

Through the use of RFID technology, can enhance the accuracy and efficiency of the work, and greatly improve the quality of service and reduce operating costs, saving labor and inventory reading space. At the same time, it can also reduce the loss of the goods in the whole logistics, such as the wrong goods, wrong delivery, theft, damage and inventory, shipping errors and so on. RFID solutions provide accurate information about the inventory situation, managers can quickly identify and correct the low efficiency of the operation, so as to achieve rapid delivery and minimize the cost of storage.

\section{Summary}

The paper presents building intelligent precise logistics service system by key technologies of RFID in the Internet of things. The intelligent logistics, optimizing the allocation of resources, to logistics enterprise business processes, and provide value-added logistics services to the end user, expand the 
scope of business, and finally realize the profit maximization. At the same time, the information exchange between them using the unified format, no matter where in the world, any company can read any object identification, and can be read or get the vector contains information. Carrier of object identification and information is the RFID tags, sensors and other low cost or embedded devices.

\section{Acknowledgements}

This paper is supported by Science and technology project of Henan Province (162102310478).

\section{References}

[1] Ren Weide, Zhang Junhu, Wang Jianping, "Design of a RFID Location Algorithm based on LANDMARC", IJACT, Vol. 5, No. 9, pp. 577 583, 2013.

[2] Seung-Jung Shin, Jung-Hwa Kang, and Kae-Dal Kwack. A Study of a Protocol Model for Information Security of the RFID Base Individual Identification Device, RNIS, Volume 7, pp. 10 12, 2011.

[3] Zhen Wu, Jin Peng, "A Chance-constrained Model of Logistics Distribution Center Location under Uncertain Environment", AISS, Vol. 6, No. 3, pp. 33 42, 2014.

[4] Joseph K. Siror, Liang Guangun, Pang Kaifang, Sheng Huanye, Wang Dong. Impact of RFID Technology on Tracking of Export Goods in Kenya, JCIT, Vol. 5, No. 9, pp. 190 199, 2010.

[5] Lvqing Yang, Caili Wang, Wenhua Zeng, "Research of Matrix-based Grouping Method on Anti-collision Algorithm for RFID Tag Identification", AISS, Vol. 5, No. 13, pp. 126 133, 2013.

[6] Joseph K. Siror, Liang Guanqun, Kaifang Pang, Sheng Huanye, Wang Dong. Use of RFID for Intelligent Pre-shipment Inspection, JDCTA, Vol. 4, No. 8, pp. $242 \sim$ 251, 2010.

[7] Keke Chen, Guohong Gao, "Using Agent and Swarm Platform to Construct Logistics Simulation System", JCIT, Vol. 8, No. 5, pp. $454 \sim$ 463, 2013.

[8] Du Jin. Application of "Internet of Things" in Electronic Commerce, JDCTA, Vol. 6, No. 8, pp. $222 \sim 230,2012$.

[9] Wei Lifeng, Ji Jianwei , Xu Jiawang. Research on the Production Logistics Information Terminal Based on RFID Technology, JDCTA, Vol. 6, No. 20, pp. $114 \sim$ 121, 2012.

[10] Min Je Cho, Hyung Rim Choi, Byung-Kwon Park, Kangbae Lee, Soon Goo Hong, Chang Hyun Park, Gwang Rok Kim. A Disaster Prevention System in the Transportation of Dangerous Goods by Using IP-RFID, JDCTA, Vol. 6, No. 1, pp. 384 390, 2012. 